\title{
Run-time and Atomic Weaving of Distributed Aspects
}

\author{
Eddy Truyen and Wouter Joosen \\ Dept. of Computer Science, K.U.Leuven, \\ Celestijnenlaan 200A, 3001 Leuven, Belgium \\ \{Eddy.Truyen, Wouter. Joosen\}@cs.kuleuven. be
}

\begin{abstract}
Run-time weaving of distributed aspects, if performed without any support for atomicity, endangers the global behavioral integrity of the application. Existing aspect-oriented middleware supports runtime weaving of distributed aspects, without addressing this problem. This inherently limits the type of behavioral changes that can be performed at run-time. This paper presents a model and an architecture for middleware, named Lasagne, that supports run-time weaving of distributed aspects in an atomic way. The paper makes the case that runtime weaving of distributed aspects is well suited for supporting dynamic and behavioral adaptations that are cross-component, cross-node or cross-layer. Adding support for atomic weaving ensures that such system-wide adaptations are performed in a safe and coordinated way.
\end{abstract}

Key words: aspect-oriented middleware, run-time weaving

\section{Introduction}

The environments in which distributed software applications must execute have become very dynamic and heterogeneous. As a result, software must be dynamically adapted or even be able to adapt itself. This is for instance especially the case in ubiquitous computing environments and environments in which the availability of resources is variable and unanticipated. The types of adaptations that should be supported are very broad. Many of these adaptations require intrusive modification of multiple classes and components if one has to rely purely on state-of-the-art software development technologies - i.e. object-oriented and component based software engineering.

Aspect-oriented software development (AOSD) tackles this shortcoming by focusing on the systematic identification, modularization, representation and composition of (often non-functional) concerns - requirements - throughout the entire software development process. Three core concepts in AOSD are concerns, aspects and weaving. In the context of this paper, it is sufficient to introduce these concepts at the level of AOP (Aspect-Oriented Programming)[24]. Concerns are similar to requirements in a broad sense of the word, ranging from high-level requirements that are articulated in an early stage of the software project ${ }^{1}$, to

\footnotetext{
${ }^{1}$ For instance, "the application should ensure confidentiality when information is exchanged between two parties".
} 
additional - often detailed - requirements that are generated when performing detailed design and implementation ${ }^{2}$. A concern corresponds in that sense with a feature, capability or quality-of-service level that is important for a stakeholder in the software project. At the programming level, an aspect is a modular unit that implements a concern. An aspect definition contains (a) behavior (code that must be executed) which is called advice and (b) a specification that expresses when, where and how to invoke the advice; this specification is called a pointcut. A pointcut is conceptually defined as a predicate that evaluates over join points. A join point is a well-defined place in the structure or execution flow of a program where additional behavior can be attached. Finally, weaving is the process of composing core functionality modules (typically application components) with aspects, thereby yielding a working system.

We observe that AOSD - when successfully supported - can simplify adaptations that emerge from changing concerns/requirements. This observation will be clarified in a motivation section (Section 2). Based on this view, we consider the dynamic adaptation process of a distributed software application as a continuous process in which aspects are woven to (and unwoven from) the distributed application at run-time in order to accommodate evolving requirements.

Many - if not most - non-functional concerns in distributed applications are distributed aspects. A distributed aspect is an aspect of which the behavior is woven (into multiple application components that are deployed) on different computer nodes of the distributed system. Aspect-oriented middleware (e.g. $[39,15,42])(\mathrm{AOM})$ supports the creation, deployment and execution of aspects for a distributed environment.

Weaving or unweaving of a distributed aspect at run-time implies adding (or removing) mutually dependent functionalities at different nodes of a distributed system. A coordination mechanism is required to preserve the behavioral integrity of the distributed application while the weaving is in progress. In fact run-time (un)weaving must be performed atomically from the perspective of the unaffected parts of the distributed application. Atomicity means that the mutually dependent functionalities must be added or removed in an all or nothing fashion.

Existing aspect-oriented middleware lacks appropriate support for atomic weaving of distributed aspects. While some systems such as Prose[41] provide support for atomic weaving of aspects within the boundaries of a single computer node, there exists to our knowledge no system that supports atomic weaving of distributed aspects. A naive approach to circumvent this problem is to suspend the execution of the distributed application until the weaving of the aspect code is completed. This approach is non-trivial, however, and incurs a service disruption which is undesirable for mission-critical applications that must permanently be available.

The main contribution of this paper is a model and an architecture for middleware, named Lasagne, that supports atomic weaving without requiring any service disruption. The design of the Lasagne middleware is essentially based

\footnotetext{
${ }^{2}$ For instance, "decrypted messages should never be cached".
} 
on two guiding principles. First, weaving of distributed aspects is performed in two phases: a deployment phase and an activation phase. Secondly, we create a solution that preserves the essence of an aspect-oriented execution environment: the atomic weaving mechanism enables a coordinated manipulation of control flow across the boundaries of different computer nodes. We will argue that the application of these two key principles provides the basis for an elegant solution that supports run-time and atomic weaving of distributed aspects.

Previous publications about Lasagne focussed on a generic model for run-time composition of behavioral extensions in component-based applications, but these publications did not integrate that model with aspect-oriented constructs. In [52], the composition model has been presented in the context of a wrapper-based programming model[16,5], while the focus in [51] was to illustrate the benefits of the composition model in a service-oriented context. This paper integrates the composition model with aspect-oriented constructs, such as pointcut and advice, yielding a run-time aspect weaving model. Furthermore it discusses how this runtime weaving model is realized in state-of-the-art aspect-oriented middleware, and how the resulting mechanisms can be usefully applied in a more general middleware context. Also, the previous publications addressed system support that allows different concurrent customizations of the application to co-exist, whereas this paper focusses on support for atomicity.

The rest of this paper is structured as follows. In Section 2 we articulate the scope and problem statement of this paper. Section 3 describes the run-time weaving model of the Lasagne middleware. Section 4 presents the architecture and design of the Lasagne middleware. We further illustrate the value of our solution in Section 5 by discussing the implementation, the applicability, performance trade-offs of Lasagne, and integration of Lasagne in standard middleware. We describe related work in Section 6 and we conclude in Section 7.

\section{Scope and Motivation}

This section first illustrates the range of adaptations that Lasagne aims to support and motivates why support for run-time aspect weaving will become an important feature of the future middleware. Subsequently the atomic weaving problem is revisited into more detail.

\subsection{Scope of Supported Adaptations}

Distributed software applications must be dynamically adapted and be able to self-adapt. We give an example to illustrate the versatility of the spectrum of adaptations. Consider an e-finance application that initially supports a limited service of delivering statements of the customer's checking and savings accounts. ${ }^{3}$

\footnotetext{
${ }^{3}$ We will sketch the evolution of this minimal service towards a fully fledged e-banking service. This illustration is based on our experience with real life experience with that type of applications - in that sense, the sketched evolution matches the real history of an application. More info can be found in [27].
} 
An initial level of security for this straightforward and minimal application could deliver confidentiality by sending the messages over an encrypted network layer - the encryption or decryption facilities will obviously be implemented in the lower layer of the middleware architecture. Security risks will increase when online transactions are supported by an extended set of services. This will be even more the case when private banking services are added, for instance to support the e-trading of securities. The security measures that will be added to the application are no longer embedded in the middleware, in fact specific security measures will be added to the application code, to use advanced authorization for financial transactions, and to add a non-repudiation feature for specific types of transactions (typically with a higher risk level). Both the financial institution and the clients may wish to have a solid audit trail that effectively enables to prove that certain transactions have been ordered by the client and executed by the bank. As the service becomes more successful and the state-of-the-art of security technologies evolves, the bank may have to adapt the cryptographic building blocks that produce signatures (for instance when certain types of hashing functions become too weak to avoid the algorithms to be broken). Also, authentication tokens do evolve; sometimes different user types may choose for different tokens (smart cards that store X.509 certificates, vs. popular generators of one-time passwords, etc.). This picture includes a lot of extensions and alternatives for an evolving security solution. It therefore illustrates that adaptations can be

- embedded in the middleware or tightly coupled to application code (E.g. IP level encryption vs. application level authorization for transactions);

- extending the existing functionality (e.g. adding signature verification through interaction with the certificate revocation list of a public key infrastructure), or replacing certain parts (e.g. when updating the signature generation techniques);

- context sensitive: user type and possibly the type of application functionality that is requested may determine the set (or one specific instance) of authentication techniques that are required.

We clearly envision a broad spectrum of adaptations. The common denominator for all these adaptations is that they are all behavioral, i.e. the adaptations require functional extensions and/or replacements, and they all target serviceoriented applications. We do not address the management of globally consistent state. Web services, for example, make as many tiers as possible state-less, and incorporate the complete state in a back-end database or in an XML document that is part of the message. It is therefore assumed that a distributed application operates according to a state-less session model, i.e. no external state dependencies towards invoking clients are engaged. Neither do we focus on adaptations that are limited to the modification of configuration parameters (e.g. QoS parameters).

We aim for a generic solution that can cover a broad spectrum of adaptations as introduced above. We believe that the concept of aspects is well suited for modelling entities that are subject to such complex behavioral adaptations. More 
importantly, the need for a behavioral adaptation occurs not only at load-time but also at run-time, hence the need for run-time weaving. For example, for signature verification multiple alternative algorithms exist and each algorithm can be implemented as a separate aspect. Which algorithm is to be applied cannot always be determined in advance. For example, when a drop of computing resources in the operating system layer occurs, the signature verification aspect in the system may have to be replaced with an alternative aspect that reduces the use of computing resources (at the price of some quality levels). Run-time weaving is required as this kind of decisions can clearly only be taken at run-time.

In order to give more safety to the run-time weaving process, our work assumes an aspect-component model where aspects have to respect the well-defined interfaces of the application components. In this way we avoid undocument coupling between aspects and application components. As such, pointcuts may only evaluate over non-invasive join points: join points which are visible in the interface of the components (typically: the declared required and provided operations and the invocation/execution of these operations). Furthermore, an aspect-component model typically keeps the behavior of an aspect reusable, by having pointcuts separately specified in an aspect binding. Consequently, behavioral adaptations can be represented by aspects that have the properties of components, enabling independent reuse.

\subsection{Distributed Aspects and Atomic Weaving}

Run-time weaving or unweaving of distributed aspects may endanger the global behavioral integrity of the system. A pedagogical illustration can be based on a simple example of encryption/decryption. Suppose a distributed producerconsumer application, in which a producer object produces data items that are sent over the network to multiple consumer objects that process the data. Now suppose that at a particular point of time, at the node of the producer the need for encrypting the messages is detected due to environmental changes. ${ }^{4}$ As such, the advice for encrypting messages must be dynamically woven into the producer object. It is clear that this single, local weaving is not enough because the encryption advice depends on the associated decryption advice that is to be composed at the nodes of the consumers. Encryption and decryption must therefore be woven in an atomic fashion to preserve behavioral integrity. Notice that we have not yet discussed atomic aspect replacement. This is a reconfiguration operation that dynamically replaces a specific aspect with another aspect by unweaving the former and weaving the latter. Obviously, such a runtime replacement must be performed in an atomic manner as well.

Appropriate coordination mechanisms for atomic weaving, unweaving and replacement are non-trivial in a distributed system. For example, in the encryption/decryption example, atomic weaving does not mean that encryption and

\footnotetext{
4 This can for instance be triggered by an intrusion detection system - techniques for detecting environmental changes and correlating them into adaptation decisions are obviously out of the scope of this paper.
} 
decryption advices must be injected at exactly the same point of time. At the point of time when the encryption advice is executed for the first time at the producer node, there may still be non-encrypted messages circulating somewhere in the network that still have to be processed by consumer objects. If an attempt to decrypt a non-encrypted message will result in an error, then the decryption advice at the consumer's nodes cannot be woven at the same time as the encryption advice at the producer's node. This atomicity problem becomes even worse when the need and types of encryption changes frequently: this is typically the case when context-sensitive adaptations have to be supported.

\section{A Model of Run-time and Atomic Aspect Weaving}

Lasagne is based on a run-time weaving model that is divided into two phases. First, aspects are deployed into the distributed application. Once deployed, aspects can be dynamically activated on a per message basis. We refer to the former mechanism as deployment and to the latter as activation. The above separation between deployment and activation allows us to apply the 'divide and conquer' principle to the complex problem of atomic weaving.

The remainder of this section is structured as follows. A high-level overview of the run-time weaving model is presented in Section 3.1. Then, the models of deployment and activation are respectively presented in Section 3.2 and Section 3.3. Subsequently, Section 3.4 summarizes our solution approach to the atomic weaving problem.

\subsection{Roles and Scenarios}

We give an overview of what are the main human actors involved and the scenarios in which these human actors interact with the Lasagne middleware.

Deployment is performed by a human operator, referred to as application deployer, who is trusted within the organization and system infrastructure boundaries of the distributed application. The application deployer uses a deployment tool to deploy, un-deploy or replace aspects, at load-time or run-time. How an aspect must be bound with a particular application, is specified by the application deployer in an aspect binding. This aspect binding basically encapsulates one or more pointcuts for the advices of the aspect. Figure 1 gives an example of an aspect binding in the context of the aforementioned producer-consumer application and the encryption-decryption aspect.

In the spirit of context-sensitive adaptation, activation (or deactivation) of an aspect is requested by a contextual actor. Contextual actors are software programs or devices, that often are based on interaction with human actors such as end users, system administrators, and domain experts (e.g. security experts who decide on the policies for access control). We describe two simple

pedagogical examples of contextual actors and explain how contextual actors are able to activate aspect-based adaptations. 


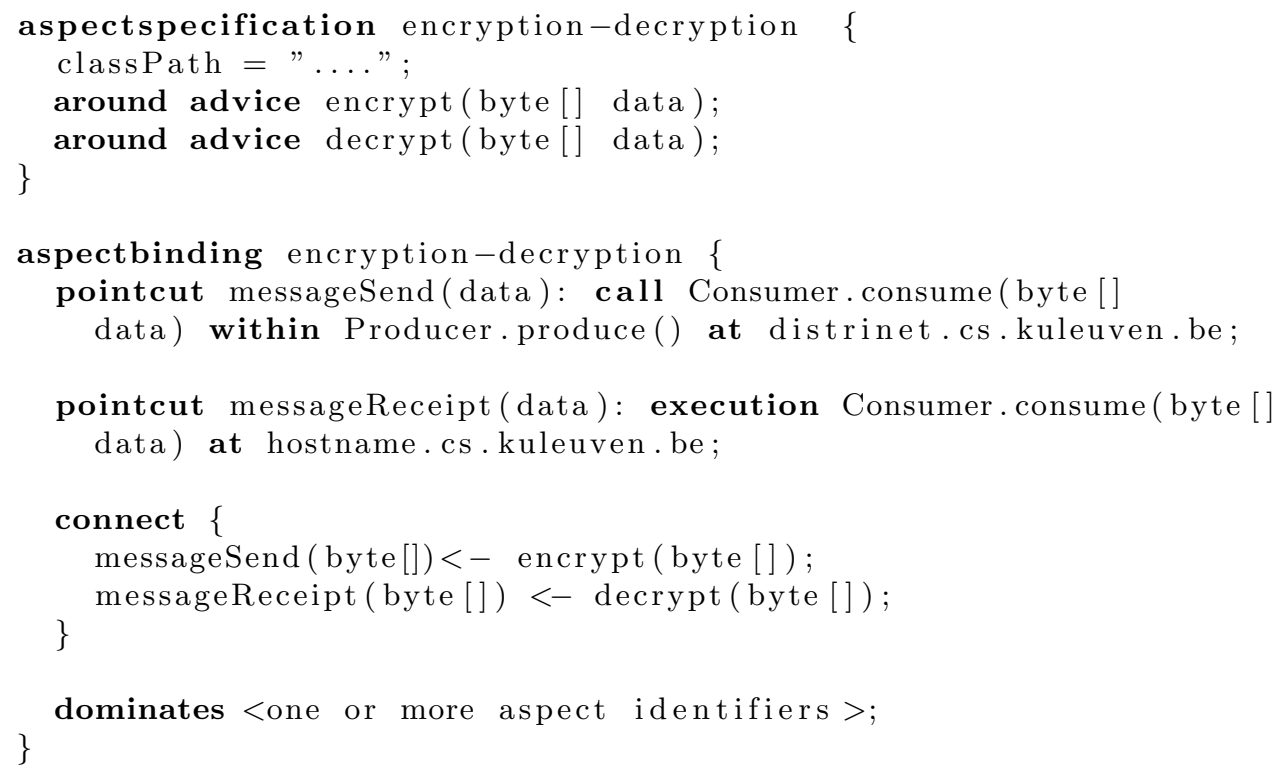

Fig. 1. The specification of the encryption-decryption aspect and its binding to the producer-consumer application is shown. The aspect specification describes the signatures of the advice methods of the aspect and contains the location where the aspect code can be downloaded. The aspect binding encapsulates one or more pointcuts and connects these pointcuts to the advices. For example, pointcut messageSend() connects the encrypt ( ) advice to a call of the consume() operation from within any Producer object that is located on host distrinet. Optionally, ordering constraints with respect to other aspects can be specified using a dominates construct, very similarly to Aspect/J.

A first example of a contextual actor is a client application or integrated device that uses the services of the aforementioned e-finance application. The client uses activation as a mechanism to customize the service to its individual preferences. Simply by tagging so called aspect identifiers to its client requests, the client is able to activate aspects that contribute functional or non-functional extensions to the e-finance application that are desired by that client. Another example of a contextual actor, is a resource monitor system component, that monitors the availability of resources in the underlying distributed system infrastructure of the e-finance application. The resource monitor uses activation as a mechanism to adapt the e-finance application to undesired fluctuations in the resource availability or to system overload. For example, during system overload, the resource monitor could deactivate a resource-intensive aspect, and instead activate a cheaper variant of the aspect by tagging all client requests, that arrive at the server-side of the e-finance application, with the aspect identifier of the cheaper aspect. 


\subsection{Deployment}

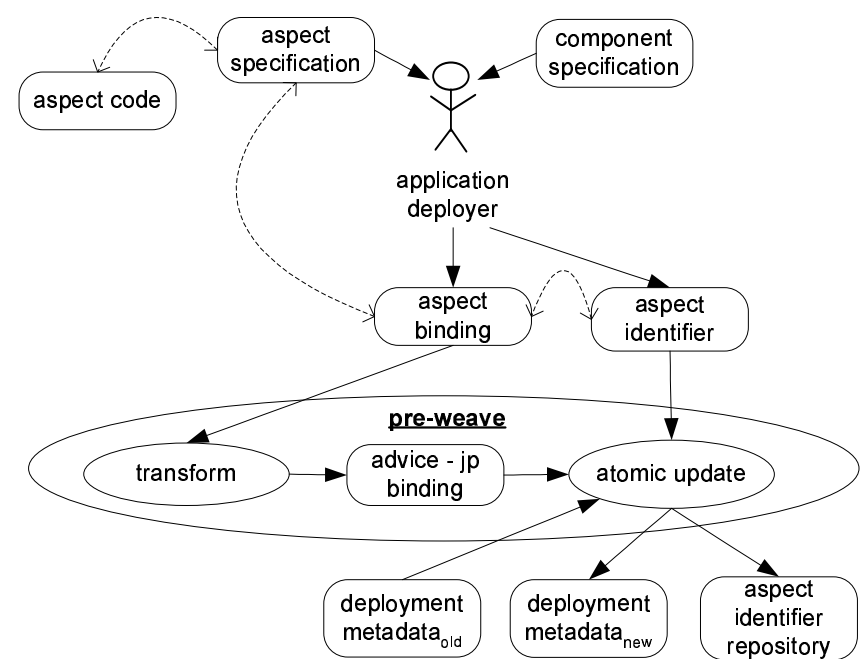

Legend
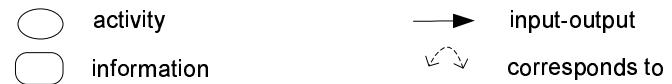

Fig. 2. Deployment data flow. The application deployer gives to the deployment tool an aspect identifier and an aspect binding. The deployment tool first transforms the aspect binding into lower-level advice-to-joinpoints bindings and then atomically updates the deployment metadata with these bindings and the corresponding aspect identifier. The aspect identifier is stored in a publicly accessible aspect identifier repository.

Figure 2 gives an overview of the deployment model of Lasagne by showing the sequence of steps that are performed to deploy a particular aspect. First, the application deployer must of course specify an aspect binding. The application deployer uses subsequently the Deployment API of the middleware to deploy the aspect. For example, the encryption-decryption binding of Figure 1 is deployed as follows:

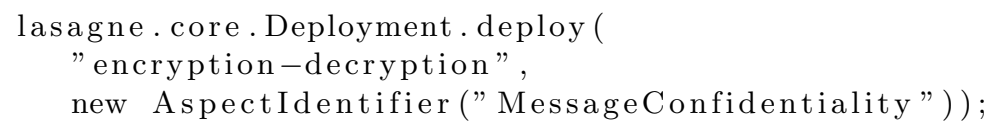

The application deployer must assign a unique name to the aspect. This name, referred to as aspect identifier, is supposed to associate with the aspect a meaning that makes sense to contextual actors. The aspect identifier may for instance describe a capability, feature or quality-of-service-level that is of interest to contextual actors. In the above example, the application deployer maps the 
encryption-decryption aspect to the aspect identifier "MessageConfidentiality" to indicate that this aspect offers a specific security property when transmitting messages.

Subsequently, the Lasagne middleware interprets the aspect binding to effectuate a so called pre-weaving of the aspect with the application. This means that the aspect is not woven directly into the application code, but it is woven into a meta-level representation of the application code, which is called deployment metadata. Deployment metadata describes a lower-level configuration of the aspect bindings in terms of which advices of aspects must be woven at which join points of application components. As shown in Figure 2, the process of pre-weaving is a consecutive process of transforming the aspect binding into a sequence of so called advice-to-joinpoint bindings, and atomically updating the deployment metadata with these bindings. An advice-to-joinpoint binding connects a specific advice to a specific join point. More specifically, it is a tuple of 4 members: the signature of the advice method, the aspect identifier associated with that advice method, the join point where the advice must be executed, and the order of execution (relative to other advices at that join point).

Finally, the aspect identifier is stored in a public aspect identifier repository for future reference on behalf of contextual actors: they need it in order to activate the aspect.

\subsection{Activation}

Deployment of an aspect does not involve "turning the switch" that effectively injects the aspect code into execution space. Instead, turning the switch is under control of flow-dependent activation that allows to initiate the execution of aspects by tagging aspect identifiers to messages.

To support multiple concurrent adaptations[49, chptr. 2] by contextual actors, activation of an aspect will only affect the current execution context (i.e. the current message flow or invocation path in which the contextual actor is involved) and will not interfere with other ongoing flows of messages. It is therefore also called flow-dependent activation.

Figure 3 gives an overview of the activation model. To request the activation of an aspect at a certain execution point, a contextual actor intercepts the application-level message that corresponds with this execution point and tags it with the desired aspect identifier. Of course, the contextual actor can tag multiple aspect identifiers to a message. Multiple aspects are thus combined and activated for the same message. Once the aspect identifiers are tagged with the message, they propagate with the invocation path of messages, succeeding the original message. As such, the aspect activation logic travels (as a set of aspect identifiers) with the message flow. The runtime weaver of the Lasagne middleware consults the tagged aspect identifiers in order to know which advices to execute for a given message. When an aspect is activated for the first time, and therefore, the code of the aspect is not yet woven into the application code, the runtime weaver has to consult the deployment metadata in order to know which advice must be woven at which join points. 


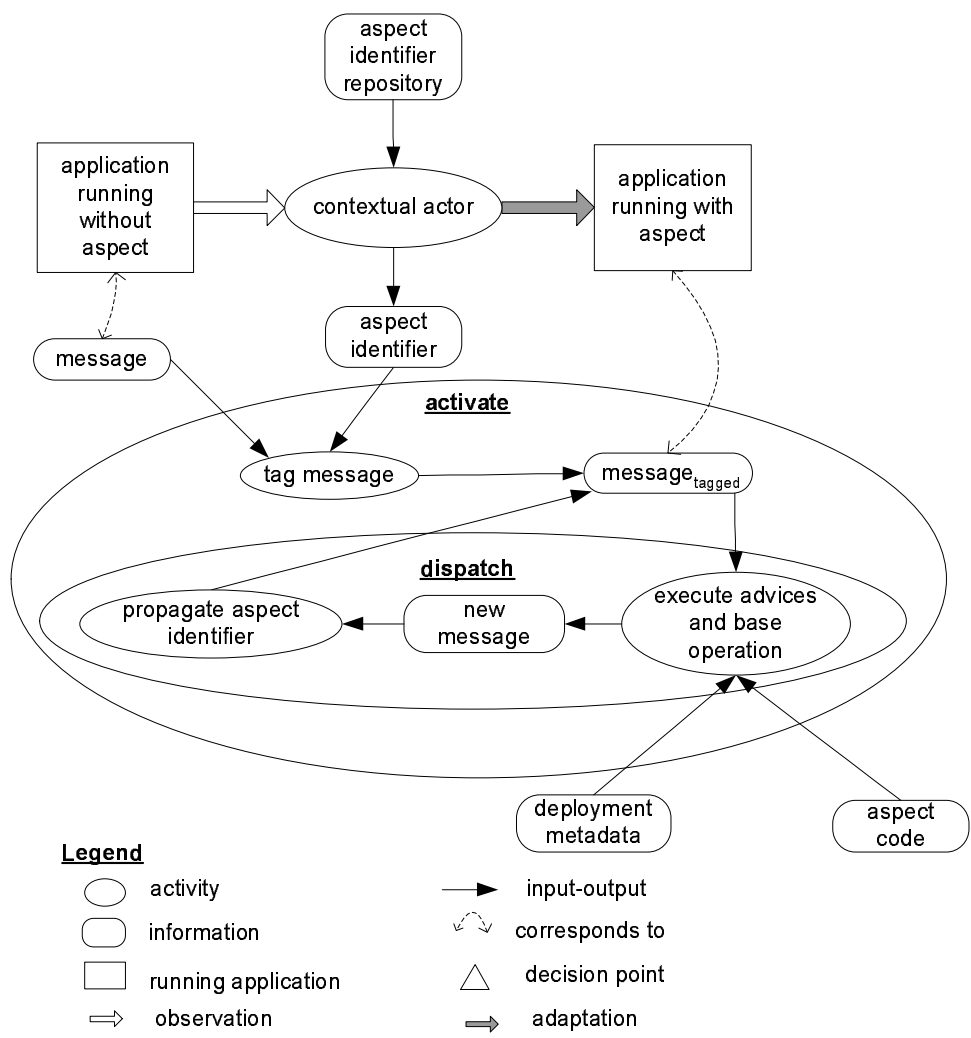

Fig. 3. Activation data flow. The contextual actor observes directly or indirectly the behavior of the application and decides at a certain point in time to adapt the application by activating one or more aspects. To activate an aspect, the contextual actor dynamically tags aspect identifiers to messages which are on their way to the application. When processing a message, the runtime weaver consults the tagged aspect identifiers in order to know which advices to execute. If some aspect is not yet injected into the application, the runtime weaver first loads the aspect's code, and updates its internal data structures, based on the deployment metadata. New messages, resulting from the original message's execution, are tagged with the same set of aspect identifiers by the runtime weaver. As such, aspect identifiers propagate. 


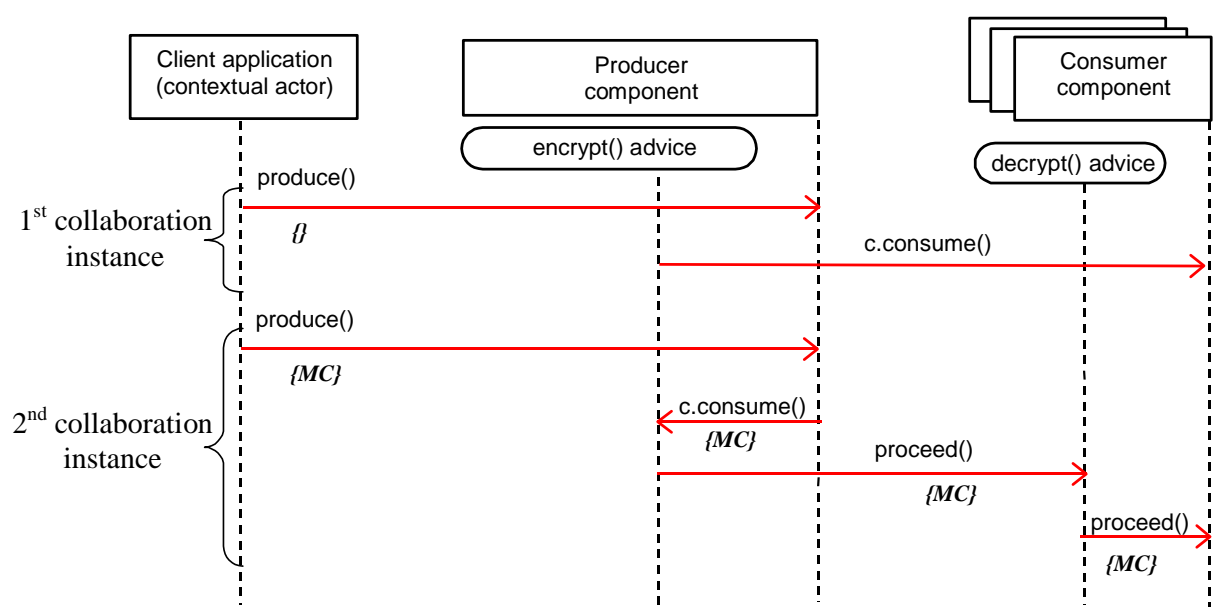

abbrevations of aspect identifiers

$0 \quad$ no aspect identifiers are tagged to the message

\{MC aspect identifier "MessageConfidentiality" is

Fig. 4. Flow-dependent activation of aspects

Figure 4 presents a message sequence diagram that illustrates the flowdependent activation of the encryption-decryption aspect in the producer-consumer example. Here a client application, using the service of the producerconsumer application, plays the role of contextual actor. Message exchange between the client application and the front-end of the producer-consumer application is based on a well-defined collaboration. (i.e. invocation of an object-based interface $)^{5}$ Client application and front-end typically perform multiple collaboration instances sequentially. For example in Figure 4 one collaboration instance has completed its execution, and a second one is running till completion. The figure illustrates that once the client application has tagged the aspect identifier "MessageConfidentiality" to the message that starts the second collaboration instance, the aspect identifier is propagated with the subsequent message flow across the producer-consumer application such that the runtime weaver knows it has to invoke the advices of the corresponding aspect along this message flow.

To manage flow-dependent activations in an effective way, it is useful to group them into sessions. A session is defined as the group of messages that are exchanged between a client and a service as part of one or more collaboration instances. The activation policy of a session, or session policy in short, is defined as the set of aspect identifiers that is activated for that particular session. In this view a flow-dependent activation can be considered as an update

${ }^{5}$ Packet-based communication using a transport protocol is another example of a collaboration that is situated at the level of the lowest middleware layer. 
of a particular session policy. Typically, the session initiator defines a particular activation policy and this policy remains the same during the whole session. Concurrently running sessions can have different activation policies. Finally, it is possible to dynamically update the activation policy of an ongoing session provided the application architecture conforms to a well-defined structural property. This property will be explained in more detail in Section 4.1.

\subsection{Summary of Atomic Weaving}

The division of the weaving process into a deployment and an activation phase enables us to decompose the problem of atomic weaving into three sub-problems that are easier to solve on their own.

Complete and correct deployment Lasagne ensures that an update to the deployment metadata due the pre-weaving of an aspect is correctly and completely distributed across all computer nodes on top of which the distributed application executes.

Consistency in the activation of aspects During flow-dependent activation, Lasagne guarantees the consistency in the activation of aspects: once a combination of aspects has been activated for a collaboration instance by tagging aspect identifiers to the message that starts that collaboration instance, this decision is consistently coordinated all over the subsequent execution of the collaboration instance by means of propagating the aspect identifiers with the message flow.

Coordination between deployment and activation An important correlation between deployment and activation is that Lasagne only permits the activation of an aspect after the aspect is completely and correctly deployed. Thus, a successful completion of the deployment protocol is a prerequisite for initiating the activation of the aspect. The underlying rationale is of course that when the binding of an aspect is incompletely represented in deployment metadata, the execution of the aspect will also be incomplete, and thus results into inconsistencies in the system-wide behavior of the application. This phased transition from deployment and activation is realized by means of a coordination protocol.

In the next section we describe the architecture and design of the Lasagne middleware with a focus on how to achieve the above three requirements. Before proceeding, we would like to point out that the division into deployment and activation also helps to improve other important properties including security, availability, scalability, etc. With respect to security, the division implies a division of authority: only the trusted application deployer is allowed to deploy new aspects. This is a first line of defense against malicious contextual actors[51]. Furthermore, as already argued, without a separate deployment phase, one would have to tolerate a temporary service disruption. Also, a separate deployment phase makes the weaving process scalable towards complex collaborations and federated applications. Suppose one wants to atomically weave multiple mutually depending sub-aspects into a large-scale federated application, where each 
sub-aspect is to be woven in a specific subsystem of the application. To do this, one first deploys the sub-aspects with the same aspect identifier, then one activates the whole configuration of sub-aspects using that unique aspect identifier. A more detailed treatment of these issues can be found in [49, chptrs. 2, 5].

\section{Architecture of the Lasagne Middleware}

In this section we present the architecture and design of the Lasagne middleware. First we give an overview of the distributed systems architecture of Lasagne. Then we elaborate upon the approach taken to achieve atomic weaving. Finally we focus on the design of Lasagne's runtime weaver which is the core of the Lasagne middleware.

\subsection{Distributed System Architecture}

The Lasagne middleware is compatible with a broad range of application architectures and platform architectures. There are only a few assumptions. With respect to the application architecture, it is assumed that the components of the distributed application are grouped in several operating system processes that are possibly allocated on different computing nodes of the distributed system. With respect to the platform, we assume that intra-process as well as interprocess communication between components is based on messages.

Figure 5 gives an overview of the distributed system architecture of the Lasagne middleware. It is conceptually divided into a deployment system and a runtime system. The deployment system implements the pre-weave activity (see Figure 2), while the runtime system implements the activate activity (see Figure 3). The deployment metadata, which is stored in a deployment metadata repository, is shared info between the deployment system and the runtime system. The deployment metadata repository may be centralized or replicated, depending on how local one wants to keep the deployment metadata to the runtime system instances, and depending on the size and topology of the distributed system.

The architecture of the deployment system consists of a deployment tool and a data tier. The deployment tool offers the functionality for transforming an aspect binding into advice-to-joinpoint bindings and for atomically updating the deployment metadata repository with these bindings. The data tier conceptually consists of three kinds of data stores. These are the aspect identifier repository (introduced in Section 3.2), a code repository that stores all the binaries of aspect and component code, and a specification repository thats acts as a back-end for the deployment tool by storing aspect bindings, and aspect and component specifications. Similar to the deployment metadata repository, several alternative architectures are possible for the data tier.

The architecture of the runtime system consists of a runtime weaver and an activator. A separate instance of the runtime weaver is running within each 


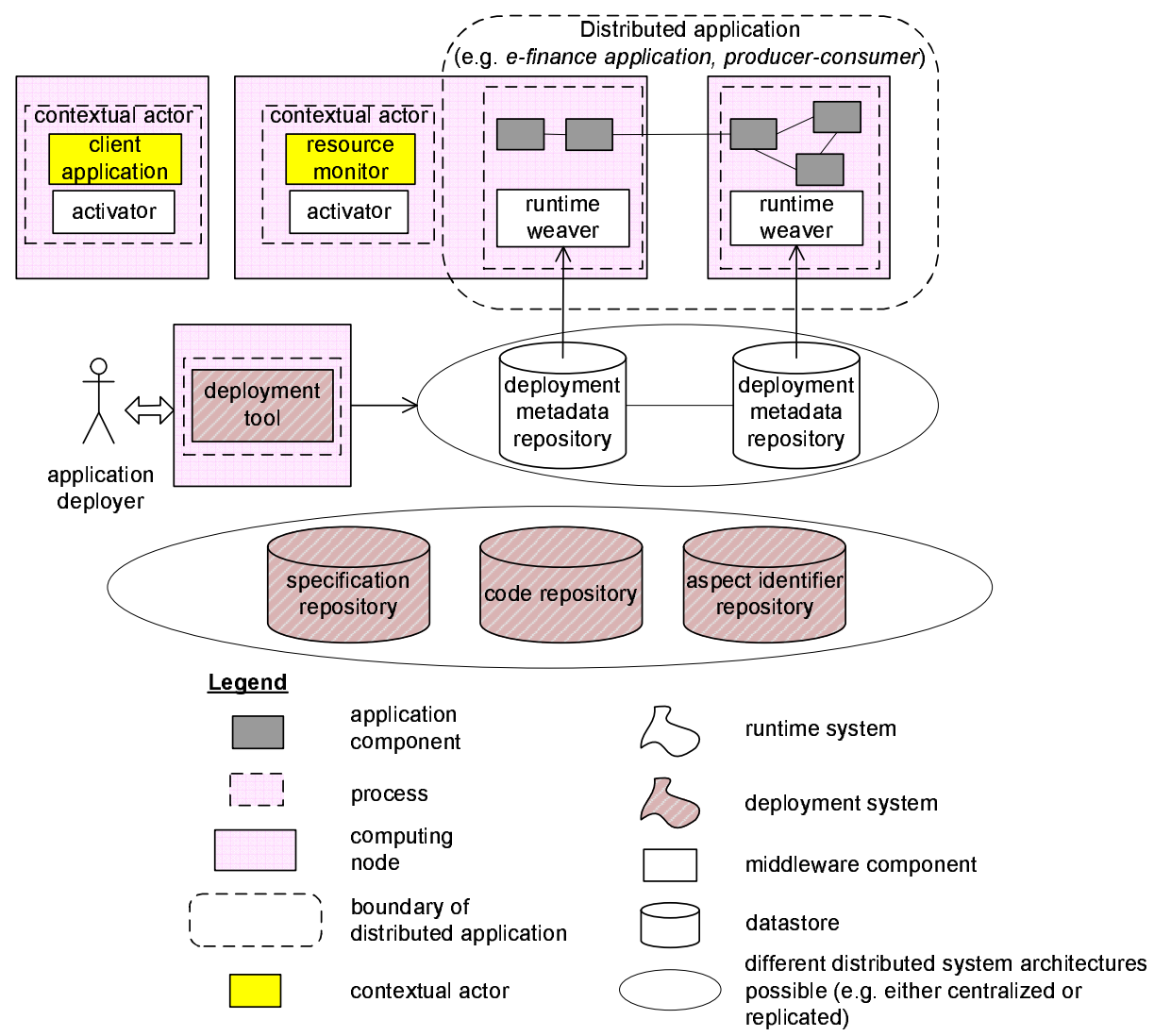

Fig. 5. Distributed system architecture of the Lasagne middleware.

process. ${ }^{6}$ Its main responsibility is to support the dispatch activity, as sketched in Figure 3. The activator component is a plug-in for contextual actors that provides support for the tag message activity in Figure 3.

Messages in Lasagne have an associated invocation context, in which properties about the ongoing computation can be stored. This invocation context is also used to tag aspect identifiers to messages. The activator component provides an interface to contextual actors for setting aspect identifiers to this invocation context field. Propagation of the aspect identifiers with the message flow is simply achieved by copying the invocation context from one message to another. The aspect identifiers are used by the runtime weaver to dynamically dispatch messages to a selected subset of advices. How this is implemented will be further clarified in Section 4.3.

${ }^{6}$ For example, in Java-based applications[3], there would be a single instance of the runtime system per Java virtual machine. 
Finally, we relate the application architecture to the notion of session policy. To ensure consistency in the activation of aspects, the activation policy of an ongoing session may only be dynamically updated at the start of a new collaboration instance. As will be explained in Section 4.2, the Lasagne middleware has the capacity to enforce this constraint for application architectures where an exterior client process (e.g. the client of the e-finance application) or any intermediate proxies (e.g. the resource monitor) require dynamic adaptations of the interior server processes (the e-finance application itself). On the other side, for application architectures where the boundary between the exterior and interior components cannot be drawn clearly ${ }^{7}$, the activation policy of a session must remain the same for the lifetime of the session. Notice that in these applications, it still is possible for concurrent sessions to have different activation policies.

\subsection{Support for Atomic Weaving}

This section presents the detailed approach of how the Lasagne middleware supports atomic weaving. As stated in Section 3.4, we have divided this complex problem into three subproblems. We describe the solution for each of these problems.

Correct and complete deployment. In order to enable correct and complete deployment of an aspect, atomicity and concurrency control must be provided at the level of updates to deployment metadata. The use of a conventional transaction protocol is the best way to implement this. Hence, we refer to the process of updating the deployment metadata of a distributed application as a deployment transaction. How to implement the deployment transaction depends on whether the deployment metadata repository is replicated or not.

A centralized repository is simply implemented as a transactional file service or database that employs a two-phase locking protocol to serialize concurrent updates.

In a decentralized approach, deployment metadata is replicated at multiple nodes of the distributed application. Here, an update of deployment metadata must be coordinated by means of a two-phase commit protocol. Concurrency control within each replica is again provided by implementing it as a transactional file service. The advantage of the decentralized approach is that the Lasagne runtime weaver can consult the deployment metadata locally.

Finally, a hierarchical approach is useful to make deployment scalable towards complex collaborations in federated applications. This hierarchical approach implies a delegation of deployment authority: different subsystems of a federated application are controlled by separate application deployers who are themselves organized in a hierarchy. Correct and complete deployment of mutually depending aspects into these different subsystems is then coordinated at the root of this hierarchy by means of a nested two-phase commit protocol.

\footnotetext{
${ }^{7}$ One can argue that such applications have a weak architecture, but this is out of the scope of our discussion.
} 
Consistency in the Activation of Aspects. As explained in Section 3.3, the core mechanism for ensuring consistency is the propagation of aspect identifiers with the message flow. As a result, within a distributed application, the runtime weaver instances in different processes are implicitly coordinated by the tagged aspect identifiers. In other words, when two runtime weaver instances are requested to dispatch messages which logically belong to the same message flow, then these messages will be consistently dispatched to the same combination of aspects.

A second element related to activation consistency implies that flow-dependent activations are only permitted for messages that start a new collaboration instance. Since we assume a state-less session model and an application architecture where the boundary between the exterior and interior components can be drawn clearly, this means that flow-dependent activations are only permitted for messages that enter the application architecture.

This constraint can be enforced in two ways: by rigorous design of the application architecture, or by run-time checks at the level of the middleware. At the design level of the application architecture, one can enforce the constraint by placing contextual actors at the right places of the application architecture. For example, every application that provides a service to client applications has some process that acts as a front-end. It is clear that tagging of aspect identifiers may only occur on messages that are exchanged between the client applications and this front-end (either at the client-side or at the server-side).

The second approach is to ensure that the underlying middleware infrastructure can automatically detect if messages enter the application architecture. We introduce the concept of an application identifier which is a specialization of the concept of aspect identifier. An application identifier is defined as a name that identifies the components that are part of the application architecture. For example, the components of the e-finance application could all be tagged with the application identifier "E-Finance", whereas the client application could be tagged with the application identifier "Client". Thus the concept of a message entering the application architecture is modelled as a message of which the sender and receiver component are associated to different application identifiers. ${ }^{8}$

Coordination between Deployment and Activation. We have separated weaving into a pre-weave and an activate activity to cope with the complexity of atomic weaving. We now bring these two activities into synchrony to effectively gain the desired atomicity property. To achieve this synchrony, a coordination protocol must be defined between the different system components of the Lasagne middleware.

Three different coordination protocols are actually required, respectively for weaving, unweaving and replacement of aspects at run-time. We now discuss the coordination protocol for aspect weaving by means of a concrete adaptation scenario.

\footnotetext{
${ }^{8}$ In practice, application identifiers are marshalled into the sender and receiver fields of the wired messages such that the activator component can verify this property.
} 
Suppose, a specific client application, which is already using the services of the distributed application, desires a feature that is not yet deployed in the application. To accommodate this request, the application deployer should deploy at run-time a new aspect that implements the feature as required by the client. ${ }^{9}$ The application deployer requests the deployment tool to deploy the aspect and hereby he associates a new aspect identifier, say $F$ to the aspect. After that, the coordination protocol for governing the actual run-time weaving of the aspect begins. Figure 6 indicates, by means of numbered arrows, what are the steps involved in this coordination protocol.

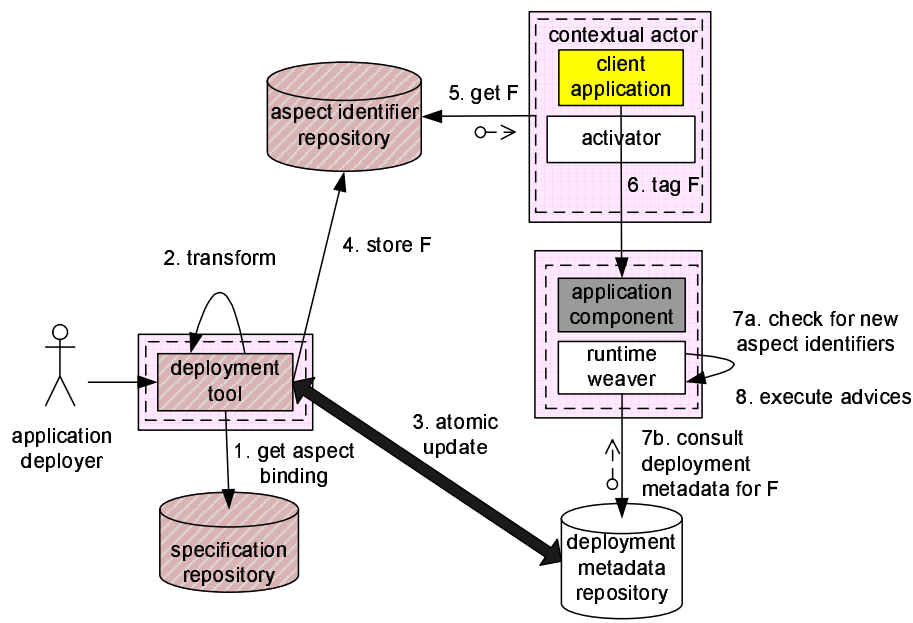

Legend (see legend of figure 5 for explanation of other symbols)

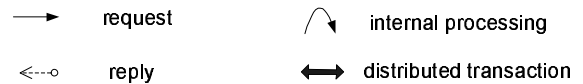

Scenario: the application deployer requests the deployment tool to deploy a specific aspect with aspect identifier $\mathrm{F}$ and, after that, a client application wants to activate this aspect.

Fig. 6. Coordination protocol for aspect weaving at run-time

The coordination protocol consists of a deployment phase and an activation phase. The deployment phase consists of 4 steps:

1. the deployment tool retrieves the aspect binding of the aspect from the specification repository,

\footnotetext{
${ }^{9}$ We assume there is an off-line meeting about the requirements of the feature between the stakeholders of the client application and the distributed application. After that, the application deployer seeks to realize the feature by means of a suitable aspect implementation and specifies the binding of the aspect with the distributed application by means of an aspect binding.
} 
2. the deployment tool transforms the aspect binding into advice-to-joinpoint bindings,

3. the deployment tool updates the deployment metadata repository with the advice-to-jointpoint bindings in a deployment transaction,

4. the deployment tool inserts the aspect identifier $F$ into the aspect identifier repository.

The activation phase consists of 4 steps:

5. the client application retrieves the aspect identifier $F$ from the aspect identifier repository,

6. the client application tags aspect identifier $F$ to its subsequent client requests,

7. the runtime weaver notices the new aspect identifier $F$ on the first arrived message and consults the deployment metadata,

8. the runtime weaver updates its internal data structures to manage the new aspect and executes the appropriate advices, related to $F$.

Obviously, the coordination protocol is very simple. This is the result of the fact that deployment and activation are quite orthogonal operations, and that the Lasagne runtime system already incorporates dedicated support for maintaining consistency in the activation of aspects.

The details of the coordination protocol for aspect unweaving and replacement can be respectively found in appendices A and B.

\subsection{Design of the Runtime Weaver}

The run-time weaver is designed as a message-based dispatcher. What is required is (a) the aspect behavior needs to be supported in the dispatch (method) tables of all application objects involved, (b) and the aspect behavior needs to be executed whenever the message (that needs to be processed) expresses the need for that particular behavior. This is non-trivial compared to a centralized application where this behavior is localized in one context: then inconsistency cannot occur. In a distributed application however, the distributed context needs to be updated without the risk of inconsistencies to occur.

We do need a mechanism as lightweight as possible. Different from most AO middleware systems, we want to support per-instance aspect-composition: this means that either dispatch tables will be created per object (which is very expensive), or dispatch tables will be shared by objects in a single process. This is a more economical approach. The behavior in such a table then is a superset of what can be executed in a specific collaboration. In this implementation context, deployment means binding specific advice behavior to distributed objects, activation means selectively executing that advice behavior. Thus, per instance run-time aspect weaving in a distributed context will be performed by inserting the required advices into the dispatch table and by executing (activating) that behavior on a per-message basis. 


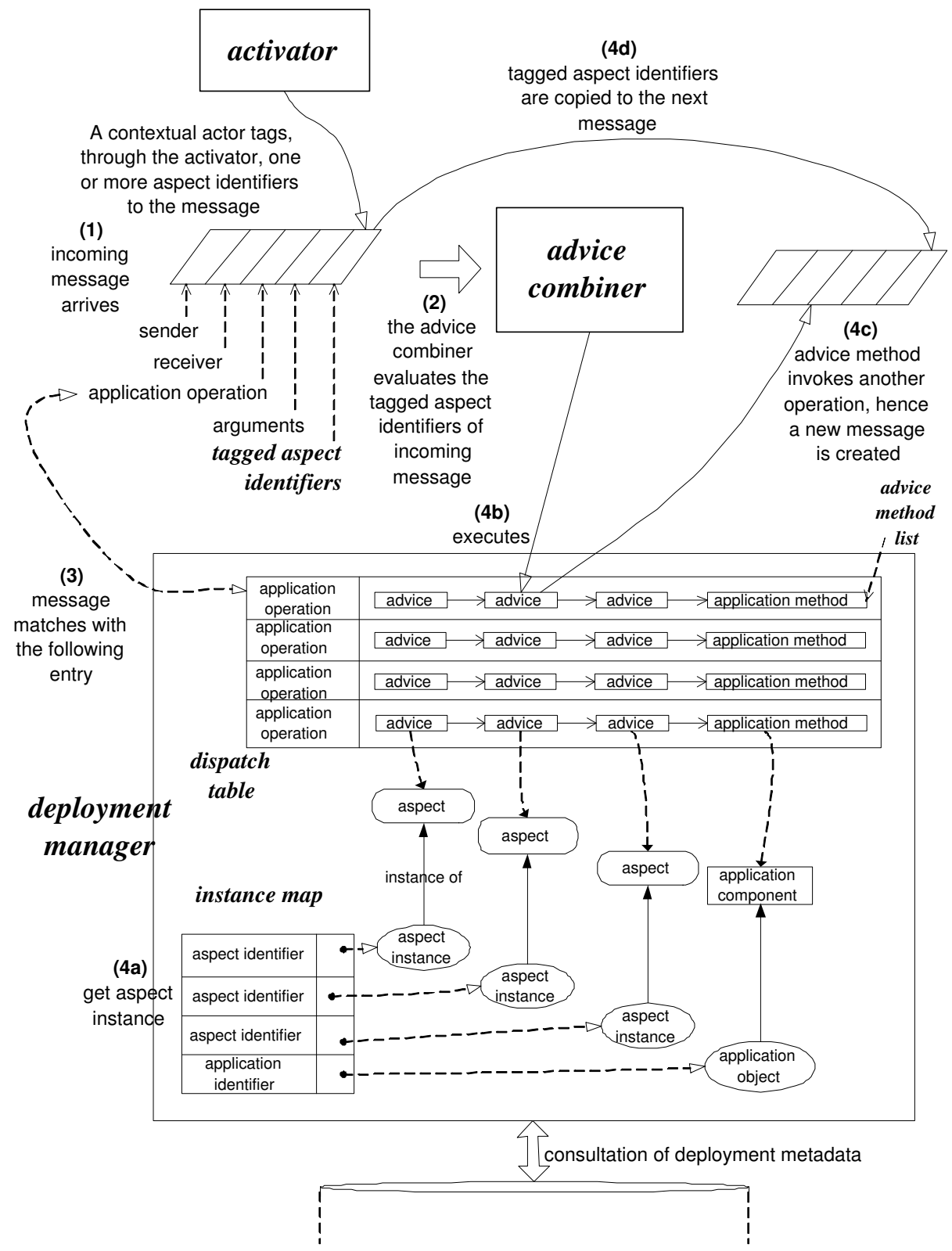

Fig. 7. The design of the run-time weaver: interplay between the advice combiner and the deployment manager 
The internal structure of the runtime weaver is exactly decomposed according to this division: as shown in Figure 7 it consists of a deployment manager component that manages the behavior (and state) of the aspects, and an advice combiner that selectively executes that behavior. As indicated above, the deployment manager maintains a dispatch table that has an entry for each provided and required operation of the application components. Each entry stores an ordered list of pointers to advice methods. The deployment manager also creates and manages aspect instances. Since a full treatment of Lasagne's aspect state management is out of the scope of this paper, it suffices to say that Lasagne supports a per-object aspect instantiation scope. More details about Lasagne's instance management can be found in [49, chptr. 5].

Figure 7 illustrates in more detail how the deployment manager and the advice combiner interact among each other to perform the actual weaving. An activator, shown at the top left corner of the figure, tags one or more aspect identifiers to a particular client request. The message flow, that represents the processing of this client request, propagates through the application. The advice combiner interprets the tagged aspect identifiers of each message and will only execute the advices associated to these aspect identifiers. For every message, the advice combiner thus selectively combines advices from the superset in the dispatch table.

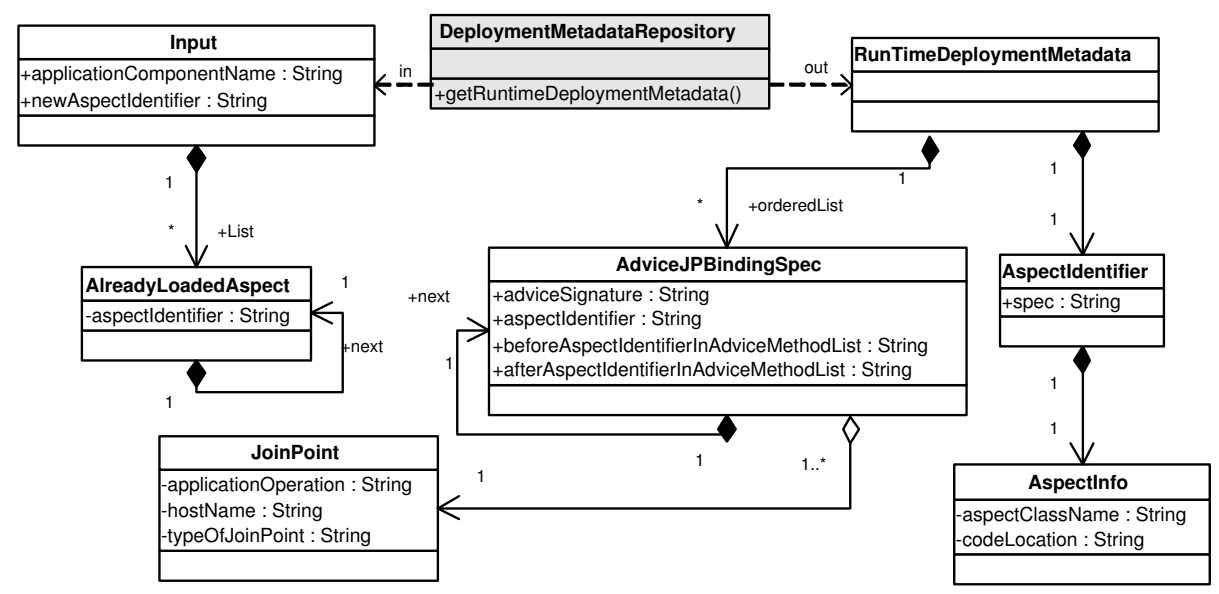

Fig. 8. Deployment metadata repository interface for consulting deployment metadata at run-time.

Possibly, the behavior related to a requested aspect identifier is not yet supported in the dispatch table. In this case, the deployment manager component first consults the deployment metadata repository for this aspect identifier. The interface of the deployment metadata repository that supports this consultation operation is shown in Figure 8. The returned deployment metadata is essentially 
a list of advice-to-joinpoint bindings and the aspect's code base location. Based on this information, the deployment manager is able to load the aspect code and insert advice method pointers into the dispatch table.

Of course, statically deployed aspects ${ }^{10}$ will be processed by the deployment manager component at load-time, using another interface of the deployment metadata repository (see appendix $\mathrm{C}$ ).

\section{Discussion}

The discussion of this work will focus on four topics: the techniques used for implementing Lasagne, the integration of Lasagne on top of standard middleware platforms, the performance trade-offs in the current design of Lasagne, and the applicability of Lasagne.

\subsection{Implementation}

To validate our run-time weaving model we have implemented a prototype of the Lasagne middleware on top of the programming language Correlate[43].

Correlate is a concurrent object-oriented language with an execution environment that makes it easier to develop distributed applications. The execution environment is designed as a meta-level architecture that offers a so called Meta-Object-Protocol (MOP)[23]. Correlate's MOP essentially offers several meta-objects that allow to inspect and modify the internal semantics of the language and execution environment. Correlate is implemented as an extension of the Java programming language[3].

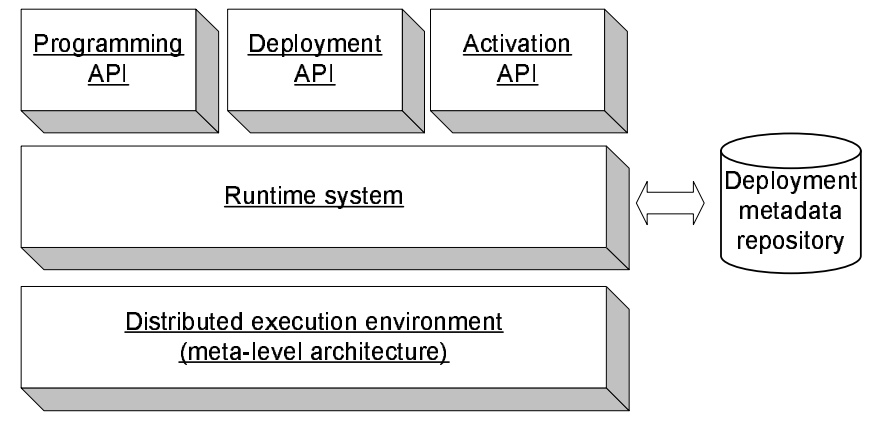

Fig. 9. Overview of the Lasagne middleware

Figure 9 illustrates the implementation structure of the Lasagne middleware. As shown in the figure it consists of three layers: the API layer that offers

${ }^{10}$ i.e., the application deployer also has the option to deploy aspects before the application is started up. 
three separate programming interfaces for programming, deploying and activating aspects; the runtime system (the core of the Lasagne middleware); and a distributed execution environment that comes with Correlate.

The runtime system of Lasagne is implemented as a modular plug-in of Correlate, using Correlate's MOP. The design of the runtime weaver has already been presented in Section 4. The activator component is implemented using Correlate's MOP as well, by relying on the particular meta-object that allows to intercept method invocations and executions as first-class message objects. The interface of the Activator component corresponds to the Activation API. The deployment metadata repository is implemented as a set of files that are managed in a distributed file system. ${ }^{11}$ Each file stores the deployment metadata of a given application component.

The Programming and Deployment API provide basic support for programming and deploying aspects. We do not require any extensions to state-of-the-art pointcut constructs. Runtime deployment is currently supported by manually updating the deployment metadata files. As outlined in Section 4.2, we only need to rely on basic transaction protocols to ensure atomicity and concurrency control when updating the deployment metadata files.

\subsection{Integration in State-of-the-art Platforms}

We compare Lasagne middleware to standards middleware such as OMG's CORBA, Enterprise Java Beans (EJB), and Microsoft .NET, and discuss how Lasagne can be integrated in these platforms.

The Lasagne runtime weaver is situated at the same level as the Portable Object Adapter(POA) component in the OMG CORBA ORB model[53], or at the same level as the Container architecture in the Enterprise Java Beans (EJB) specification[34] and the OMG CORBA Component model[54]. An important difference is however, that in traditional object-based middleware, message dispatch (or similar mechanism in container-based middleware) is only driven by the receiver's object identity that is stored in the message. In Lasagne, message dispatch is (in addition) driven by aspect identifiers.

The tagging of aspect identifiers can be commonly achieved in CORBA by means of portable interceptors[37] or in .NET by means of a special API that gives access to the logical call context[28].

In Lasagne, aspect identifiers are propagated with the message flow by storing the identifiers as part of the reified messages, and copying them along the invocation path. Another approach is storing the aspect identifiers in a thread local variable. We have not followed this approach in order to circumvent the problem of losing logical thread identity when control crosses system boundaries, or when control is asynchronously handed over between two threads as in the active object model[36]. Some of the standard middleware platforms already provide a mechanism to cope with this problem such as distributed threads[10] in real-time CORBA or the LogicalCallContext class in .NET[28].

\footnotetext{
${ }^{11}$ UNIX Sun OS 5.8 implementation of the Sun network file system[44].
} 
Finally, the aspect identifier repository can be compared with a standard component repository, with the difference that it enables lookup of (distributed) aspects, whereas a standard component service enables lookup of (distributed) components.

\subsection{On Performance Trade-Offs}

The proposed model and architecture has clearly been focused on providing generic support for a broad range of behavioral adaptations that have to be performed in a distributed system. Notice that we aimed for a solution that enables different context-dependent versions of the application to coexist, and that should enable distributed service offering without any disruption because of adaptations. It therefore seems obvious that we have created an architecture which generates quite some overhead in achieving the proposed objectives. We analyze the costs by addressing (1) cost in terms of service availability, (2) overhead in terms of the cost of processing interactions, (3) overhead in terms of memory consumption.

(1) Our solution offers consistent behavioral adaptation of distributed entities without causing unavailability of any service or application feature.

(2) We have chosen to obtain the value of our solution at the cost of an increased processing time for invocations. The reason is threefold:

1. We want a solution that guarantees no service disruption when an (complex) adaptation is in progress. This is an inherently solid basis for mission-critical systems that must be permanently on-line.

2. We anticipate that a broad range of network applications (e.g. the typical case of web services) is relatively insensitive to processing delays as communication delays often dominate the response times in executing distributed services.

3. From a methodological viewpoint, we believe that it is a sound strategy to ensure that (a) a first (base level) architecture is offering a robust solution with absolute guarantees (no service disruption and atomicity) and that (b) optimizations - probably weakening other qualities (e.g. flexibility) - are pursued thereafter. This is exactly the scope of our ongoing and future work. For instance, Lasagne has already an option to limit deployment of aspects to load-time only. Flexibility cuts are minor (aspects can still be dynamically activated and deactivated on a per message basis), while the performance overhead due to the coordination between deployment and activation is eliminated.

(3) Our architecture is extremely economical when it comes to memory cost. Deploying distributed aspects implies the storage of a logical reference (aspect identifier) in the nodes where a specific aspect must be executed. Pro-actively loading aspects would lead to substantially larger memory footprints, as well as extra delays. In the current solution, aspect code is loaded on demand, i.e. when the first activation of the required behavior occurs. 


\subsection{Applicability}

For several application areas, it has become increasingly more important that software can be dynamically adapted to evolving requirements of a given context. Our discussion will focus on three such application areas: application service provisioning, autonomic computing, and adaptive Quality of Service (QoS). The proliferation of web services, and recent initiatives such as Microsoft's Office Live[11] indicate that software applications are gradually evolving from a desktop model to a service provider model. For example, Microsoft Office Live will offer expert business management applications as on-line services without that their customers need Office or Windows installed on their desktop computers. Moreover, the underlying system infrastructure is not only targeted at remote application execution, but also at the uniform deployment and integration of new applications. In other words, the vision[48], that system software such as compilers will some day in the future be offered as distributed service objects, seems to emerge. This evolution creates a completely new meaning for the notion of a client request. When popular application services begin to have a large number of client request on a daily basis, it becomes economically profitable for the service provider to make such application services more dynamic and customizable to the needs of individual customers. Existing on-line (web) services are already personalized. Such personalization is however limited to the user interface and data that is offered to the customer. An additional opportunity is the supporting of customization scenarios in which customers can dynamically adapt the behavior of the application service itself. Needless to say that Lasagne is very well suited for providing this type of client-specific customizations.

Lasagne can also provide a complementary advantage to middleware support for autonomic computing and self-healing[17,7], and adaptive QoS[4]. Research in these areas is targeted towards extending middleware with policies and techniques for context monitoring and dynamic selection of adaptation strategies. To our knowledge, these middleware extensions do not automate the task of coordinating adaptation operations across multiple processes and computer nodes; the coordination must be explicitly managed by the programmer. After all, these middleware extensions are integrated with an object-based platform, thereby constraining the representation of a single adaptation strategy as inherently localized. We believe that Lasagne will be useful for automating cross-node coordination requirements in QoS control and autonomic computing systems as Lasagne offers a solid service-enabling platform for the management and coordinated selection of cross-process and cross-node adaptation strategies.

\section{Related Work}

Five categories of related work are considered: recent aspect-oriented middleware advances, network software, dynamic software architecture, coordinated adaptation in distributed systems and reflective middleware. 
Aspect-oriented middleware. The general relation to other aspect-oriented middleware platforms has already been sketched in the motivation of this paper. To our knowledge, no other aspect-oriented middleware exists to date that provides support for atomic weaving of distributed aspects. The most important difference between existing $\mathrm{AO}$ middlewares and Lasagne is that their run-time weaving model does not support the distinction between deployment and activation.

DADO [55] (distributed aspects for distributed objects) middleware offers an innovative IDL languages for programming distributed aspects in heterogeneous environments. DADO has a mechanism, called Remote Multiple Contextual Invocation (RMCI), that provides a form of dynamic per-invocation selection of aspects as in Lasagne. However this mechanism is restricted within the scope of a single client-server interaction and, therefore, does not provide any guarantees with respect to system-wide consistency in the activation of aspects.

CAM/DAOP $[15,40]$ is a component and aspect based approach that combines the benefits of both CBSD and AOSD disciplines. An innovative aspect of $\mathrm{CAM} / \mathrm{DAOP}$ is that it specifies the composition of components and aspects using an architectural description language (ADL)[45], named DAOP-ADL. This ADL-based approach provides an interesting complement to Lasagne (or any run-time weaving approach for that matter). After all, using DAOP-ADL, application deployers are able to comprehend the overall aspect-component composition, facilitating a better understanding and easier verification of the application as a whole. This is of course an important software engineering quality that improves the safety and robustness of deploying aspects at run-time.

Lasagne would, in the same vain, be nicely complemented by model-driven middleware (e.g. [18]) and aspect-oriented domain modelling (e.g. [20]). Combining these approaches leads to a very powerful concept. Design models of aspects and applications can be specified, composed and possibly verified. Once composed, these models can be automatically synthesized to deployment metadata for a specific middleware platform of choice.

Referring to our discussion on the applicability of Lasagne to adaptive QoS, Duzan et al. [14] have built an aspect-oriented middleware platform, called QuO, that allows to separate adaptive QoS concerns from the application. The middleware also provides support for coordinating adaptation operations across multiple processes and computer nodes. Lasagne could be useful for automating that coordination support.

Dynamic architectures for network protocols. Some of the problems related to ensuring consistency in the activation of aspects (see Section 4.2) have already been addressed, to a certain extent, in networking protocols. It is common for messages to have dynamically computed headers in order for peers to process the message correctly by dispatching it through a series of protocols. Also at the client-side, the shift towards dynamic protocol stack composition architectures (e.g $[35,32])$ makes that the protocol stack at the server-side must have the intelligence to deal with this correctly.

There are two important differences between Lasagne and network protocols however. First, Lasagne is more generic because it is aimed to support adap- 
tations at different layers of software, including the application layer[52], middleware layer[50] and network layer[22]. Secondly, Lasagne's support is reusable across multiple application domains. In protocol stacks the support for message dispatching is wired inside the protocol stack architecture itself, whereas in Lasagne all support is externalized in a separate runtime system that can be applied to any family of applications.

Another related technology in this field is programmable networking. Programmable networking stands for a whole spectrum of networking technologies that aim to introduce more openness and adaptability into today's networks by making network routers programmable. Historically there have been two main approaches to the provision of programmability in networks[13]: First, in the $a c$ tive networking paradigm[47] so called 'active packets' carry programs that execute on 'active' nodes. Secondly in the open signaling approach (e.g [8]), routers export 'control interfaces' through which they can be remotely (re)configured by out-of-band, application-specific, signaling protocols.

Overall, the active networking approach is the most dynamic since it enables application-specific adaptations across the network in a very fine-grained scale. However, it is perceived as more vulnerable to security treats from malicious applications, and there is also the problem of domain mismatch[31]: application programmers, who wish to customize the underlying network to their preferences, are suddenly confronted with a domain (in this case, active networks) that is completely different from their familiar application domain. The open signaling approach does not suffer from these problems since it supports adaptations that are programmed by a trusted and informed third party. However, open signaling is an order of magnitude less dynamic.

We position Lasagne in the middle of these two approaches:

- Similar to the open signaling approach, adaptations are programmed by a trusted application deployer who is an expert at his application domain (in this case, network protocols).

- Similar to the active network approach, once adaptations are deployed, they can be dynamically activated on a per message basis by contextual actors (which in this case act as the (client) applications wanting to customize the underlying network).

Adaptations in programmable networking can be more complex compared to the range of adaptations that are supported in Lasagne, but this also leaves the door open for more intrusive changes that endanger safety. A second important difference is that Lasagne is more generic. The application area of programmable networking is more like a niche, and of course, the approach is somewhat optimized for that niche.

Dynamic software architectures. In Regis[30] and ArchStudio[38], distributed applications are constructed from a number of components and connectors that encapsulate the interactions between these components. Here, the term "component" corresponds with the traditional view of component-based development. 
The emphasis of these works is on the description, dynamic reconfiguration and evolution of the application's architecture. Connectors help to decouple components from one another and the systems use configuration languages to describe the configuration of the components. The runtime structure of the application is altered by applying a program written in the configuration language to the current architecture, thus generating a different arrangement of components and connectors. Our goal differs from these works in that Lasagne focusses on adapting an application to context-specific needs, instead of coping with runtime software evolution in general. Furthermore, Lasagne's adaptation process is based on a system-wide additive refinement of existing application components with crosscutting aspects, rather than replacing existing components with new ones and switching connectors.

Coordinated adaptation in distributed systems. In the context of the above component-based reconfiguration approaches there is a whole field of research (e.g. $[26,38,19,2,9,1,21])$ that looks at the issue of how to achieve coordinated adaptation in a distributed system: when a reconfiguration involves replacing multiple components, atomicity problems appear that are very similar to those that have been studied in this paper. The existing approaches to coordinated adaptation in a component-based setting can be classified according to

- their impact on service disruption as either disruptive (e.g. [2]) or nondisruptive (e.g. [1]) approaches.

- their ability to replace an existing component by a new version or to let both be used simultaneously as either single-configuration-managed (e.g. [2]), or multiple-configurations-managed (e.g. [19]) approaches.

Lasagne clearly belongs to the multiple-configurations-managed and nondisruptive category. ${ }^{12}$ Remarkably, we have not found any coordinated adaptation approach in a component-based setting that combines non-disruptive and multiple-configurations-management. Admittedly, the problem of coordinated adaptation has been mostly studied in a setting where adaptation is triggered out-of-band, rather than in-band as Lasagne supports.

Another important difference is that some of the aforementioned approaches also employ techniques and protocols for preserving global state consistency[33] and application state invariants[2]. As stated in Section 2, Lasagne has not been designed for addressing global state consistency.

Reflective middleware. Other related work are projects (e.g. [25] and [6]) that use reflection[29] to achieve adaptive middleware. Generally, the use of reflective techniques allows for more powerful ${ }^{13}$ (i.e. intrusive) adaptations in middleware and distributed applications. However, employing reflection for context-sensitive

\footnotetext{
12 The first point may require some explanation. Lasagne offers a multipleconfiguration-managed approach because the flow-dependent activation mechanism implies that an application may be running in different versions simultaneously.

13 relative to the support of typical aspect-oriented middleware
} 
adaptations yields often a too complex system that cannot be understood by the typical contextual actor (or more specifically, the human operators involved). In this respect, Lasagne offers a very simple adaptation model to the contextual actor. For example, by simply tagging aspect identifiers to its messages, (client) applications can dynamically adapt the underlying middleware to its preferences.

More recent research on reflective middleware [12] has focussed on combining reflection with component framework technology[46] in order to preserve behavioral integrity. Much of this work has focussed on enforcing semantically compatible compositions of middleware components. Lasagne could be useful for implementing some of these integrity requirements, especially those that relate to maintaining semantic compatibility across multiple nodes.

\section{Conclusion}

This paper presents a model and an architecture for middleware that supports run-time and atomic weaving of distributed aspects without causing any disruption in the on-line availability of the distributed application. We have argued that the concept of aspects is well suited for modelling complex behavioral adaptations - i.e. not limited to the modification of configuration parameters. Furthermore, we assume that these adaptations do not introduce external state dependencies towards invoking clients.

The run-time weaving process has been divided into a deployment phase and a subsequent activation phase: an aspect is first pre-woven into so-called deployment metadata, and then the aspect is activated on a per message basis. The architecture of Lasagne documents that this division between deployment and activation creates a solution for the atomic weaving problem which includes three elements. (1) Aspects must be completely and correctly pre-woven into deployment metadata. (2) When an aspect is activated for a particular collaboration instance this decision must be consistently propagated within the subsequent message flow. (3) A simple coordination protocol acts as a synchronization barrier to ensure that activation is only possible for aspects that have been completely and correctly deployed. This approach is not only a guarantee for atomicity; the division into deployment and activation makes the run-time weaving process also more secure and scalable.

In our future work we aim to develop optimizations of the Lasagne model. We will also address atomic weaving techniques that target out-of-band adaptations and application architectures with state-full sessions.

Acknowledgments. This work has been supported by the Institute for the promotion of innovation by science and technology in Flanders.(IWT). We would like to thank the anonymous reviewers and Mario Südholt for their useful com-

ments on previous drafts. Many thanks to Nico Janssens, Bert Lagaisse and Frans Sanen for the fruitful discussions and for their feedback. 


\section{References}

1. S. Ajmani, B. Liskov, and L. Shrira. Scheduling and simulation: How to upgrade distributed systems. In Ninth Workshop on Hot Topic in Operating Systems (HotOS-IX), 2003.

2. J. P. A. Almeida, M. Wegdam, M. van Sinderen, and L. Nieuwenhuis. Transparent dynamic reconfiguration for corba. In Proceedings of the 3rd International Symposium on Distributed Objects and Applications (DOA 2001), pages 197-207. IEEE Computer Society, 2001.

3. K. Arnold and J. Gosling. The Java Programming Language. The Java Series. Addison-Wesley Publishing Company, 1996.

4. Klara Nahrstedt Baochun Li. A control-based middleware framework for quality of service adaptations. IEEE Journal of Selected Areas in Communication, Special Issue on Service Enabling Platforms, 17(9):1632-1650, September 1999.

5. D. Bäumer, D. Riehle, W. Siberski, and M. Wulf. Role object. In Pattern Language of Program Design 4, pages 15-32. Addison Wesley, 2000.

6. G. S. Blair, G. Coulson, P. Robin, and M. Papathomas. An architecture for next generation middleware. In IFIP International Conference on Distributed Systems Platforms and Open Distributed Processing, pages 191-206. Springer, 1998.

7. Gordon S. Blair, Geoff Coulson, Lynne Blair, Hector Duran-Limon, Paul Grace, Rui Moreira, and Nikos Parlavantzas. Reflection, self-awareness and self-healing in openorb. In WOSS '02: Proceedings of the first workshop on Self-healing systems, pages 9-14. ACM Press, 2002.

8. Prashant R. Chandra, Allan Fisher, Corey Kosak, T. Ng, Peter Steenkiste, Eiichi Takahashi, and Hui Zhang. Darwin: Customizable resource management for valueadded network services. In ICNP, pages 177-188, 1998.

9. W.-K. Chen, M. A. Hiltunen, and R.D. Schlichting. Constructing adaptive software in distributed systems. In Proceedings - the 21st IEEE International Conference on Distributed Computing Systems, pages 635-643. IEEE Computer Society, 2001.

10. R. Clark, D. E. Jensen, and F.D. Reynolds. An architectural overview of the alpha real-time distributed kernel. In Proceedings of the USENIX Workshop on Microkernel and Other Kernel Architectures, April 1992.

11. 2006 Microsoft Corporation. Microsoft office live. http://www.microsoft.com/office/officelive/default.mspx.

12. Geoff Coulson, Gordon S. Blair, Michael Clarke, and Nikos Parlavantzas. The design of a configurable and reconfigurable middleware platform. Distributed Computing, 15(2):109-126, 2002.

13. Geoff Coulson, Gordon S. Blair, David Hutchison, Ackbar Joolia, Kevin Lee, Jo Ueyama, Antônio Tadeu A. Gomes, and Yimin Ye. NETKIT: a software component-based approach to programmable networking. Computer Communication Review, 33(5):55-66, 2003.

14. Gary Duzan, Joseph Loyall, Richard Schantz, Richard Shapiro, and John Zinky. Building adaptive distributed applications with middleware and aspects. In AOSD '04: Proceedings of the 3rd international conference on Aspect-oriented software development, pages 66-73, New York, NY, USA, 2004. ACM Press.

15. L. Fuentes, M. Pinto, and P. Sánchez. Dynamic weaving in cam/daop: An application architecture driven approach. In Proceedings of the Dynamic Aspect Workshop in conjunction with AOSD 2005, March 2005.

16. E. Gamma, R. Helm, R. Johnson, and J. Vlissides. Design Patterns, Elements of Reusable Object-Oriented Software. Addison-Wesley, 1995. 
17. A. G. Ganek and T. A. Corbi. The dawning of the autonomic computing era. IBM System Journal, 42(1):5-18, 2003.

18. A. Gokhale, D. Schmidt, B. Natarajan, J. Gray, and N. Wang. Model-driven middleware, 2004.

19. J. Gouveia, G. Koutsoukos, L. Andrade, and J. Fiadeiro. Tool support for coordination-based software evolution. In TOOLS Europe 2001. IEEE Computer Society Press, 2001.

20. Jeff Gray, Ted Bapty, Sandeep Neema, Douglas C. Schmidt, Aniruddha Gokhale, and Balachandran Natarajan. An approach for supporting aspect-oriented domain modeling. In Proceedings of the second international conference on Generative programming and component engineering, pages 151-168. Springer-Verlag New York, Inc., 2003.

21. Nico Janssens, Wouter Joosen, and Pierre Verbaeten. NeCoMan: Middleware for Safe Distributed-Service Adaptation in Programmable Networks. IEEE Distributed Systems Online, 6(7), July 2005.

22. B. N. Jørgensen, E. Truyen, F. Matthijs, and W. Joosen. Customization of Object Request Brokers by application specific policies. In Middleware 2000, volume 1795 of Lecture Notes in Computer Science, pages 144-163. Springer, 2000.

23. G. Kiczales, J. des Riviers, and D. Bobrow. The Art of the Meta-Object Protocol. MIT Press, 1991.

24. G. Kiczales, E. Hilsdale, J. Hugunin, M. Kersten, J. Palm, and W. G. Griswold. An Overview of AspectJ. In ECOOP2001 - Object-oriented Programming, volume 2072 of Lecture Notes in Computer Science, pages 327-353. Springer, 2001.

25. F. Kon, M. Román, P. Liu, J. Mao, T. Yamane, L. C. Magalhãs, and R. Campbell. Monitoring, security, and dynamic configuration with the dynamicTAO reflective ORB. In Middleware 2000, volume 1795 of Lecture Notes in Computer Science, pages 121-143. Springer, 2000.

26. J. Kramer and J. Magee. The evolving philosopher problem: Dynamic change management. IEEE Transactions on Software Engineering, 16(11):1293-1306, 1990.

27. Bert Lagaisse, Bart De Win, and Wouter Joosen. SoBeNet: financial case study - Part 1: requirements and analysis. Report CW 404, Department of Computer Science, K.U.Leuven, Leuven, Belgium, 2005. URL = http://www.cs.kuleuven.ac.be/publicaties/rapporten/cw/CW404.abs.html.

28. J. Lowy. Contexts in .NET: Decouple components by injecting custom services into your object's interception chain. MSDN Magazine, March, 2003.

29. P. Maes. Concepts and experiments in computational reflection. In Proceedings of the Conference on Object-Oriented Programming Systems, Languages, and Applications, pages 147-155. ACM SIGPLAN Notices 22(12), 1987.

30. J. Magee, N. Dulay, and J.Kramer. Regis: A constructive development environment for distributed programs. Distributed Systems Engineering Journal, 1(5), 1994.

31. Frank Matthijs, Nico Janssens, and Pierre Verbaeten. Automatic service composition: a case for active networks usability. Report CW 356, Department of Computer Science, K.U.Leuven, Leuven, Belgium, January 2003. URL = http://www.cs.kuleuven.ac.be/publicaties/rapporten/cw/CW356.abs.html.

32. Sam Michiels, Nico Janssens, Lieven Desmet, Tom Mahieu, Wouter Joosen, and Pierre Verbaeten. A component platform for flexible protocol stacks. In Component-Based Software Development for Embedded Systems: An Overview of Current Research Trends, volume 3778/2005 of Lecture Notes in Computer Science, pages 185-208. Springer-Verlag, GmbH, November 2005.

33. K. Moazami-Goudarzi. Consistency preserving dynamic reconfiguration of distributed systems. PhD thesis, Imperial College, London, March 1999. 
34. R. Monson-Haefel. Enterprise JavaBeans, 3rd Edition. O'Reilly, September 2001.

35. Robert Morris, Eddie Kohler, John Jannotti, and M. Frans Kaashoek. The click modular router. In SOSP, pages 217-231, 1999.

36. O. Nierstrasz. Composing active objects - the next 700 concurrent object-oriented languages. In Research Directions in Concurrent Object-Oriented Programming, pages 151-171. MIT Press, 1993.

37. Object Management Group. The Common Object Request Broker: Architecture and Specification - Version 3.0, July 2003. Available at http://doc.ece.uci.edu/CORBA/formal/02-06-33.pdf.

38. P. Oreizy, N. Medvidovic, and R. N. Taylor. Architecture-based runtime software evolution. In Proceedings of the 1998 International Conference on Software Engineering, pages 177-186. IEEE Computer Society Press / ACM Press, 1998.

39. R. Pawlak, L. Seinturier, L. Duchien, and G. Florin. JAC: A flexible solution for aspect-oriented programming in Java. In Reflection 2001, volume 2192 of Lecture Notes in Computer Science, pages 1-24. Springer, 2001.

40. M. Pinto, L. Fuentes, and J.M. Troya. Daop-adl: An architecture description language for dynamic component and aspect-based development. In Proceedings of the Second International Conference on GPCE, volume 2830 of LNCS, page pp. 118137. Springer-Verlag, September 2003.

41. A. Popovici, G. Alonso, and T. Gross. Just in time aspects: Efficient dynamic weaving for Java. In Proceedings of the 2nd International Conference on AspectOriented Software Development (AOSD 2003), pages 100-109. ACM press, 2003.

42. A. Popovici, A. Frei, and G. Alonso. A proactive middleware platform for mobile computing. In Middleware 2003, volume 2672 of Lecture Notes in Computer Science, pages 455-473. Springer, 2003.

43. B. Robben. Language Technology and Metalevel Architectures for Distributed Objects. PhD thesis, Katholieke Universiteit Leuven, Belgium, 1999.

44. R. Sandberg, D. Golgberg, S. Kleiman, D. Walsh, and B. Lyon. Design and implementation of the sun network filesystem. Innovations in Internetworking, pages 379-390, 1988.

45. M. Shaw and D. Garlan. Software Architecture: Perspective on an Emerging Discipline. Prentice-Hall, 1996.

46. C. Szyperski. Component Software: Beyond Object-Oriented Programming. ACM Press and Addison-Wesley, 1998.

47. D. L. Tennenhouse, J. M. Smith, W. D. Sincoskie, D. J. Wetherall, and G. J. Minden. A survey of active network research. IEEECM, 35(1):80-86, January 1997.

48. Mario Tokoro. The society of objects. OOPS Messenger, 5(2):3-12, 1994.

49. E. Truyen. Dynamic and Context-Sensitive Composition in Distributed Systems. PhD thesis, Dept. of Computer Science, Katholieke Universiteit Leuven, Belgium, 2004.

50. E. Truyen, B. Nørregaard Jørgensen, and W. Joosen. Customization of componentbased Object Request Brokers through dynamic reconfiguration. In Technology of Object-Oriented Languages and Systems - TOOLS 33, pages 181-194. IEEE Computer Society, 2000.

51. E. Truyen, B. Vanhaute, W. Joosen, and P. Verbaeten. Consistency management in the presence of simultaneous client-specific views. In Proceedings of the International Conference on Software Maintenance (ICSM'02), pages 501-510. IEEE Computer Society, 2002. 
52. E. Truyen, B. Vanhaute, W. Joosen, P. Verbaeten, and B. Nørregaard Jørgensen. Dynamic and selective combination of extensions in component-based applications. In Proceedings of the 23rd International Conference on Software Engeneering (ICSE'01), pages 233-242. IEEE Computer Society, 2001.

53. Steve Vinoski. CORBA: Integrating diverse applications within distributed heterogeneous environments. IEEE Communications Magazine, 35(2):46-55, February 1997.

54. Nanbor Wang, Douglas C. Schmidt, and Carlos O'Ryan. Overview of the corba component model. Component-based software engineering: putting the pieces together, pages 557-571, 2001.

55. Eric Wohlstadter, Stoney Jackson, and Premkumar Devanbu. DADO: enhancing middleware to support crosscutting features in distributed, heterogeneous systems. In ICSE 2003, pages 174-186, 2003.

\section{A Coordination Protocol for Aspect Unweaving}

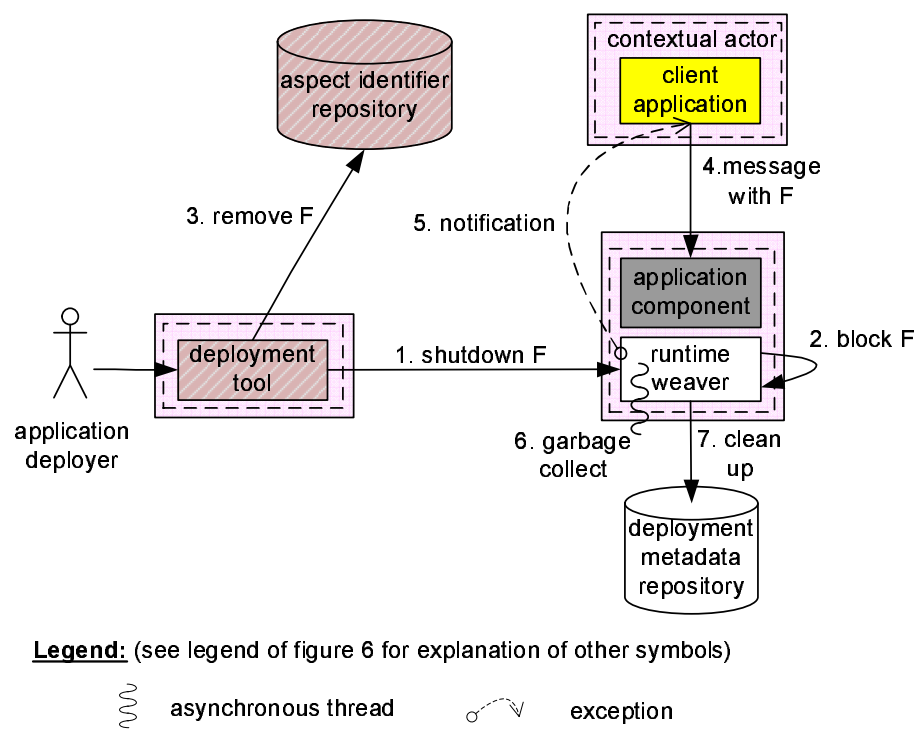

Scenario: the application deployer requests the deployment tool to unweave an aspect, with aspect identifier $F$, from the application.

Fig. 10. Coordination protocol for aspect unweaving at run-time

Figure 10 presents the coordination protocol for unweaving an aspect at runtime. Aspect unweaving is useful for adaptation scenarios where the stakeholders of the distributed application want to remove a certain aspect from the application because of various reasons, e.g. the aspect is not often activated by the contextual actors, it is malfunctioning, or it is too heavy in terms of resource 
usage. In any case, these adaptation scenarios always involve a request from the application deployer to unweave an aspect with, say, aspect identifier $F$.

The coordination protocol consists of a deactivation phase and an un-deployment phase. The deactivation phase consist of 5 steps (see Figure 10):

1. the deployment tool requests all runtime weaver instances to shutdown the execution of aspect $F$,

2. each runtime weaver instance configures itself such that it will block new messages from the outside (i.e. messages that enter the application architecture),

3. the deployment tool removes aspect identifier $F$ from the aspect identifier repository,

4. a client application sends a client request carrying the aspect identifier $F$,

5 . the client application receives a runtime exception from the runtime weaver that aspect identifier $F$ is not available anymore.

The un-deployment phase consists of 2 steps:

6. when all ongoing collaboration instances for which aspect identifier $F$ is activated have completed their execution, an asynchronously running thread will update the internal data structures of each runtime weaver instance such that all advice code and aspect instances, associated to $F$, can be released from memory,

7. each runtime weaver notifies the local deployment metadata repository, such that the latter can remove all relevant advice-to-joinpoint bindings from the deployment metadata.

\section{B Coordination Protocol for Run-time Aspect Replacement}

Figure 11 presents the coordination protocol for replacing an aspect at run-time. Replacement means that the application deployer requests the deployment tool to replace an aspect, having aspect identifier $F_{\text {old }}$, with a new aspect identifier, having aspect identifier $F_{\text {new }}$.

An important property of the coordination protocol is that replacement is performed transparent for the contextual actors that have activated the old aspect. To achieve this transparency, the second phase employs automatic aspect identifier conversion.

The coordination protocol is a combination of the coordination protocols for weaving and unweaving, and therefore consists of three phases: deploying the new aspect, deactivating the old aspect and activating the new aspect, and un-deploying the old aspect. Notice that deactivation and activation occurs in parallel. This is possible because we target a state-less session model (i.e. aspects do not engage external state dependencies with invoking clients). 


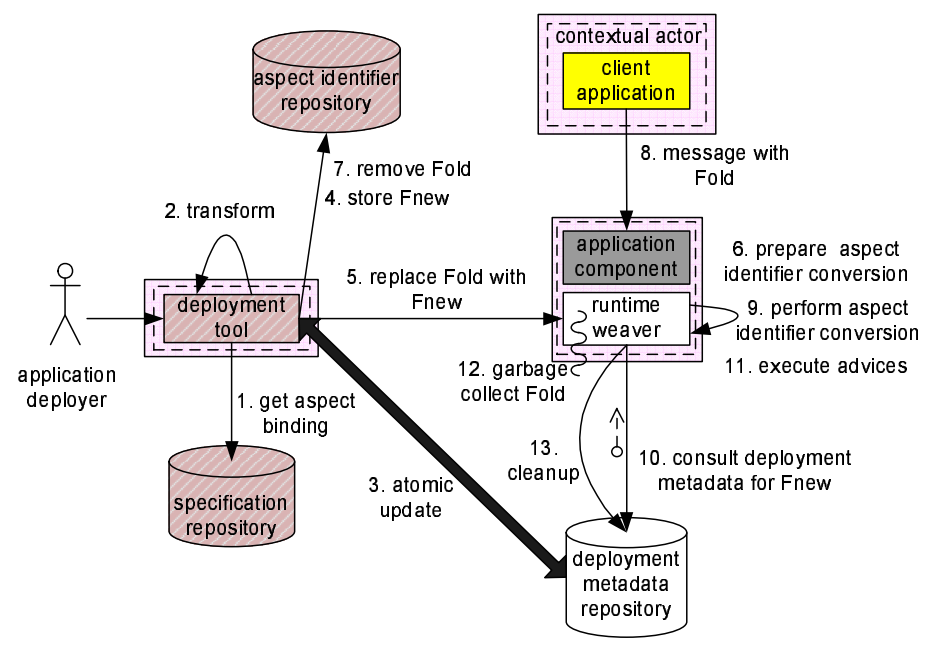

Legend: see legend of figure 10 for explanation of symbols

Scenario: the application deployer requests the deployment tool to replace an aspect, having aspect identifier Fold, with a new aspect, having aspect identifier Fnew. This should be performed transparent for the clients who have activated

F_old.

Fig. 11. Coordination protocol for aspect replacement at run-time

Deploying the new aspect. This phase consists of 5 steps (see Figure 11):

1. the deployment tools retrieves the aspect binding of the new aspect,

2. the deployment tool transforms the aspect binding into advice-to-joinpoint bindings,

3. the deployment tool updates the deployment metadata repository with the advice-to-jointpoint bindings in a deployment transaction,

4. the deployment tool inserts the aspect identifier $F_{\text {new }}$ into the aspect identifier repository,

5. the deployment tool requests all runtime weaver instances to replace aspect $F_{\text {old }}$ with aspect $F_{\text {new }}$.

Deactivating the old aspect; Activating the new aspect. This phase consists of 6 steps:

6. each runtime weaver instance configures itself such that it will transform new messages from the outside (i.e. messages that enter the application architecture) that carry the aspect identifier $F_{\text {old }}$ into carrying $F_{\text {new }}$,

7. the deployment tool removes aspect identifier $F_{\text {old }}$ from the aspect identifier repository,

8. a client request carries the aspect identifier $F_{\text {old }}$,

9. the client request is converted by the runtime weaver such that $F_{\text {old }}$ is detached from the client request and instead $F_{\text {new }}$ is tagged to it, 
10. the runtime weaver consults the deployment metadata for $F_{n e w}$,

11. the runtime weaver instance updates its internal data structures and executes the advices that are associated to $F_{n e w}$.

Un-deploying the old aspect. This phase consists of 2 steps:

12. when all ongoing collaboration instances for which aspect identifier $F_{\text {old }}$ is activated have completed their execution, an asynchronously running thread will update the internal data structures of each runtime weaver instance such that all advice code and aspect instances, associated to $F_{\text {old }}$, can be released from memory,

13. each runtime weaver instance notifies the local deployment metadata repository, so that the latter can remove all relevant advice-to-joinpoint bindings, associated to $F_{\text {old }}$, from the deployment metadata.

\section{Load-time Consultation of Deployment Metadata}

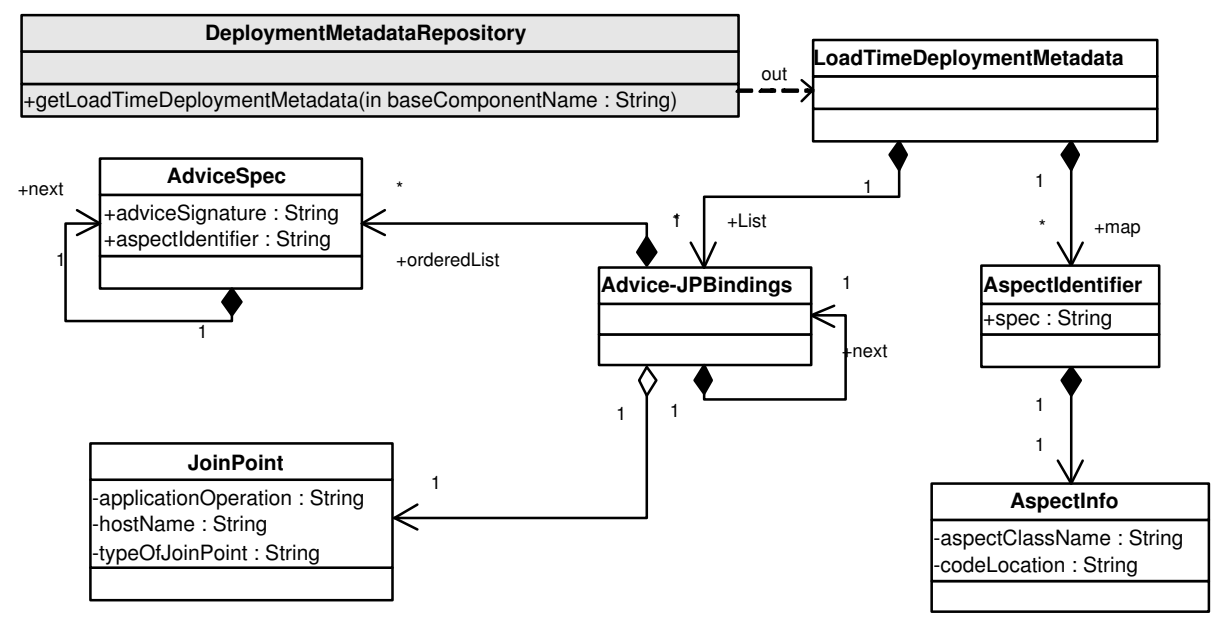

Fig. 12. Deployment metadata repository interface for consulting deployment metadata at load-time.

The specification of this operation is presented in Figure 12 as an UML model. As shown in the figure, the deployment manager receives a record structure of two elements. First, information related to where the aspects' implementations can be downloaded. Second, for every application operation, an ordered list of advice-to-joinpoint bindings is returned. 